\title{
Gastric Ulceration and Bleeding with Hemodynamic Instability Caused by an Intragastric Balloon for Weight Loss
}

\author{
Larrite Reed, Hawa Edriss and Kenneth Nugent \\ Department of Internal Medicine, Texas Tech University Health Sciences Center, Lubbock, TX, USA
}

Obesity in the United States is a medical crisis with many people attempting to lose weight with caloric restriction. Some patients choose minimally invasive weight loss solutions, such as intragastric balloon systems. These balloon systems were approved by the Federal Drug Administration (FDA) in 2015-2016 and have been considered safe, with minimal side effects. We report a patient with a two-day history of melena, abdominal pain, hypotension, and syncope which developed five months after placement of an intragastric balloon. Esophagogastroduodenoscopy with balloon removal revealed a small 8-mm gastric ulcer in the incisura. This gastric ulcer probably developed secondary to mechanical compression of the stomach mucosa by the gastric balloon which contained $900 \mathrm{~mL}$ of saline. The FDA is now investigating five deaths since 2016 associated with these second-generation balloons. Clinicians should be aware of these complications when evaluating patients with gastrointestinal complications, such as bleeding.

Clin Endosc 2018;51:584-586

Key Words: Gastric balloon; Stomach ulcer; Gastrointestinal hemorrhage; Obesity

\section{INTRODUCTION}

Obesity is an epidemic in the United States with 81 million Americans suffering from this chronic medical disease. ${ }^{1}$ Negative health effects associated with obesity include hypertension, diabetes, and many other common disorders. The intragastric balloon (IGB) system is a device used for obesity (body mass index [BMI] of $30-40 \mathrm{~kg} / \mathrm{m}^{2}$ ) treatment. At least three IGB systems have been approved by the Federal Drug Administration (FDA) in 2015-2016 for use in the United States. The IGB is either inserted endoscopically or swallowed as a capsule then filled with air or saline. These IGB systems are wide-

\footnotetext{
Received: February 18, 2018 Revised: March 20, 2018

Accepted: March 31, 2018

Correspondence: Kenneth Nugent

Department of Internal Medicine, Texas Tech University Health Sciences Center, 36014 th Street, Lubbock, TX 79430, USA

Tel: +1-806-743-6847, Fax: +1-806-743-3560, E-mail: kenneth.nugent@ttuhsc.edu ORCID: https://orcid.org/0000-0003-2781-4816
}

(c) This is an Open Access article distributed under the terms of the Creative Commons Attribution Non-Commercial License (http://creativecommons.org/ licenses/by-nc/3.0) which permits unrestricted non-commercial use, distribution, and reproduction in any medium, provided the original work is properly cited. ly used for several reasons, including effective weight loss $(10 \%-15 \%)$ over six months and the relative safety of a minimally invasive procedure. ${ }^{2}$ The older (first) generation IGB systems were withdrawn 7 years after their introduction in the market in 1985 due to serious adverse events, including gastric ulcers and perforations and intestinal obstructions. This led to the development of newer (second) generation IGB systems, mainly the ReShape and Orbera balloon systems, that are considered safer and more effective. ${ }^{2}$ Many patients have chosen IGB placement over bariatric surgery due to ease of placement and limited side effects, and there have been very few case reports describing potentially lethal side effects associated with IGB placement. ${ }^{3}$ However, there have been four recent deaths related to the Orbera system and one death related to the ReShape system; additional cases with serious side effects are being reviewed by the FDA, including, for example, gastric perforation associated with poor proton pump inhibitor compliance. ${ }^{3}$ The FDA has begun to investigate the safety of these newer second generation balloons and is recommending prompt reporting of adverse events. ${ }^{4}$ 


\section{CASE REPORT}

A 47-year-old man presented with melena, abdominal pain, and syncope for two days. He had no prior history of gastrointestinal (GI) bleeding. The patient underwent IGB (ReShape Integrated Dual Balloon System; ReShape Medical, Inc., San Clemente, CA, USA) placement with the instillation of $900 \mathrm{~mL}$ of saline for weight loss five months prior to presentation. He had no past history of alcohol consumption or liver disease. He was taking esomeprazole $40 \mathrm{mg}$ delayed release oral capsules once daily and had recently taken ibuprofen $400 \mathrm{mg}$ daily for the past five days for back pain. Vital signs included blood pressure 65/55 mm Hg, heart rate 98 beats/minute, $\mathrm{O}_{2}$ saturation $97 \%$ on room air, temperature $98.5^{\circ} \mathrm{F}$, and respiratory rate 19 breaths/minute. His pre-Rockall score was 2. His hemoglobin dropped to a nadir of $7.1 \mathrm{gm} / \mathrm{dL}$ during hospitalization from a level of $14.3 \mathrm{gm} / \mathrm{dL}$ eight months prior to admission. The patient was admitted to the medical intensive care unit due to hemodynamic instability and was resuscitated with normal saline and one unit of packed red blood cells. Abdominal computed tomography showed two connected saline-filled gastric balloons (Fig. 1). He underwent esophagogastroduodenoscopy; a small $8 \mathrm{~mm}$ gastric ulcer Forrest class III) was found in the incisura, and the balloon was removed (Fig. 2). Biopsy revealed severe chronic gastritis and Helicobacter pylori on immunoperoxidase staining. He was discharged after a five-day hospital stay.

\section{DISCUSSION}

The "REDUCE Pivotal Trial" provides important information about outcomes and complications associated with the

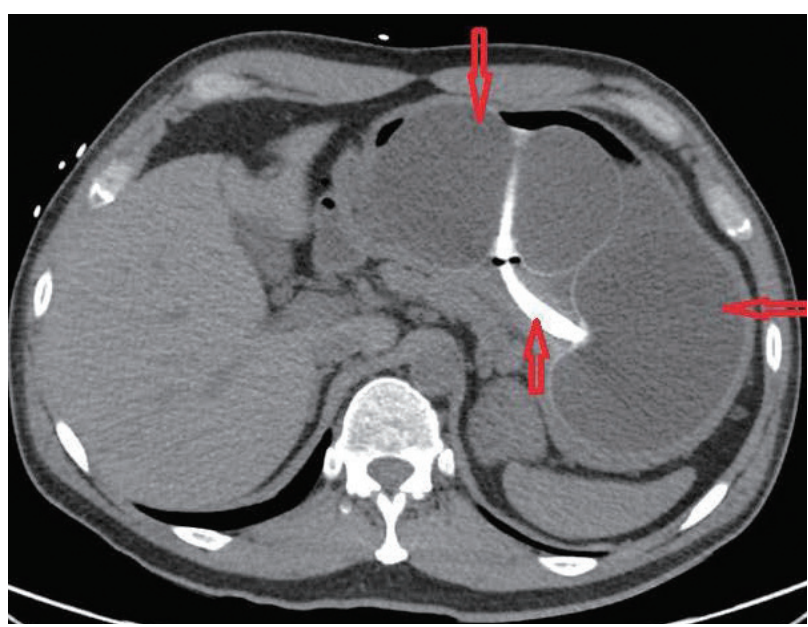

Fig. 1. Abdominal computed tomography showed two connected saline-filled gastric balloons (red arrows) in the patient. use of these devices. This trial included 326 participants between the ages of 21 to 60 years, with BMIs in the range of 30$40 \mathrm{~kg} / \mathrm{m}^{2}$ and at least one comorbid condition related to obesity. ${ }^{5}$ Participants were randomized into a control group that included diet, exercise, and a sham endoscopy or into a study group that included diet, exercise, and IGB placement for six months. The control group lost $7.8 \pm 14.1$ pounds; the study group lost $15.9 \pm 11.9$ pounds. ${ }^{5}$ Six months after removal of the device, the study group maintained $66 \%$ of the initial weight loss; this information was not reported in the control group. The Reshape IGB system requires the endoscopic implantation of a dual balloon (Fig. 2) that should conform to the stomach's natural curvature. The balloon is filled with a blue dye that serves an indicator for deflation by turning the patient's urine a blue-green color. ${ }^{5}$ After these balloons are placed, the patient is expected to follow up for six months to monitor for deflation and other possible complications. Nearly all patients in this trial developed accommodative symptoms that included nausea, vomiting, and/or abdominal pain/discomfort. These symptoms mostly resolved in 3-7 days; management included reassurance, medications, and fluid. Some subjects (9.1\%) required early retrieval due to device intolerance. There were no deaths, intestinal obstructions, gastric perforations, or device migrations during this trial. Most serious adverse events (21 of 28 patients, based on the FDA serious event definition, including requiring medical or surgical intervention) were visits to the emergency room for medical management of accommodative symptoms. Other adverse events included an esophageal mucosal tear during retrieval, requiring placement of hemostatic clips (one subject), an ulcer at the gastroesophageal junction with GI hemorrhage requiring transfusion (one sub-

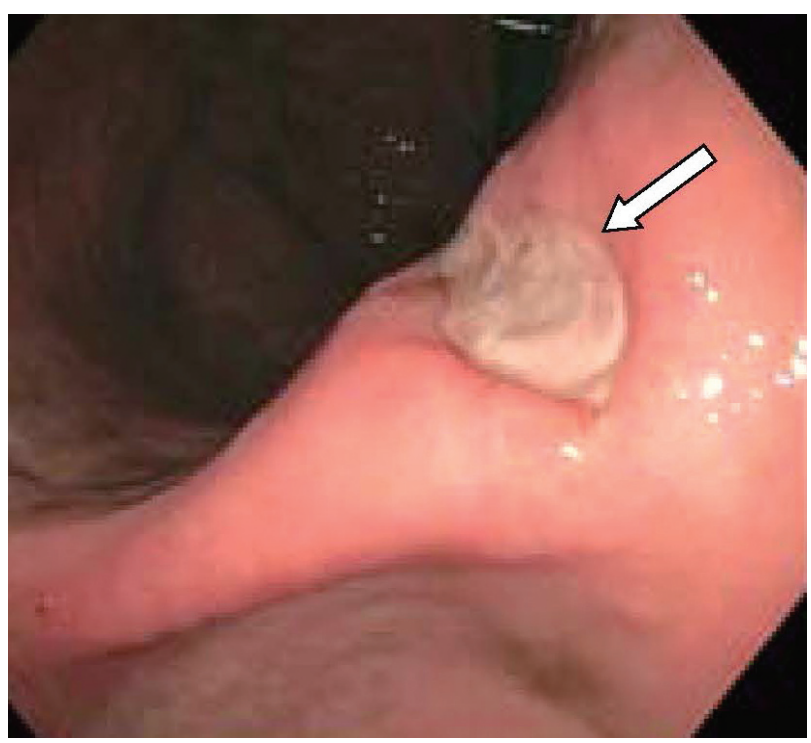

Fig. 2. Linear ulcer found near the incisura (Forrest class III). 
ject), cervical esophageal perforation requiring antibiotics during retrieval (one subject), and post retrieval pneumonitis requiring antibiotics (one subject). Gastric ulceration at or near the gastric incisura occurred in $35 \%$ of these subjects. This led to a modification of the device tip during the trial, which reduced the frequency of ulcers to $10 \%{ }^{5}$

Our case demonstrates that the newer generation balloons can cause serious complications that are probably related to persistent extrinsic pressure on the gastric mucosa. ${ }^{3}$ This patient had two risk factors for gastric bleeding (use of a nonsteroidal anti-inflammatory drug and a positive biopsy for $H$. $p y$ lori) which probably contributed to the development of this complication. He presented with significant anemia and hemodynamic instability. Yoo et al. reported a 47-year-old woman $\left(\right.$ BMI $\left.30 \mathrm{~kg} / \mathrm{m}^{2}\right)$ with an IGB placed seven weeks prior to presentation who complained of mild epigastric pain and had a gastric perforation at the base of an ulcer. ${ }^{3}$ The patient had not been taking a proton pump inhibitor, and histology studies of the ulcer revealed $H$. pylori. ${ }^{3}$ Complications such as these were frequently associated with the first generation IGBs and led to design improvements. Newer generation IGB systems are safer, but mechanical complications, such as pancreatitis, esophageal and gastric perforation, balloon migration, intestinal obstruction, and bleeding can occur. Other possible complications include malabsorption, malnutrition, and delayed gastric emptying interfering with drug pharmacokinetics.

The FDA is currently investigating five deaths since 2016 that occurred within a month of balloon placement. The FDA has not determined a root cause of death in these cases and has not established a definitive link between these deaths and the insertion procedure or the device. These complications could have resulted from surgeon error, pre-existing comorbidities (such as $H$. pylori), or spontaneous over inflation. The initial phase of the REDUCE Pivotal trial demonstrated that gastric ulcers occur frequently in these patients but usually do not cause important bleeding complications. ${ }^{5}$ Patients who undergo IGB insertion need careful follow-up post insertion and systematic reporting to determine complication rates. ${ }^{6,7}$ Additionally, these balloons should not stay in place for more than six months, and patients should be educated about regular follow up. Recently, a 28-year-old woman was lost to follow-up and presented two years after balloon placement with a small bow- el obstruction due to balloon migration that required laparoscopic surgery for removal. ${ }^{8}$ Current recommendations indicate that patients should not have an IGB system in place for more than six months, should take a proton pump inhibitor, and those with a documented history of $H$. pylori should have screening and treatment before balloon insertion. ${ }^{3,6,7}$ Additionally, patients on treatment for other comorbidities should be monitored carefully, and drug levels should be checked when indicated to ensure therapeutic levels. GI bleeding has multiple causes, but clinicians should consider these devices when evaluating patients with GI bleeding or unexplained anemia.

Conflicts of Interest

The authors have no financial conflicts of interest.

\section{Author Contributions}

Conceptualization: Larrite Reed, Hawa Edriss, Kenneth Nugent

Data curation: LR, HE, KN

Formal analysis: LR, HE, KN

Writing-original draft: LR, HE, KN

Writing-review \& editing: LR, HE, KN

\section{REFERENCES}

1. Kumar N, Sullivan S, Thompson CC. The role of endoscopic therapy in obesity management: intragastric balloons and aspiration therapy. Diabetes Metab Syndr Obes 2017;10:311-316.

2. Papademetriou M, Popov V. Intragastric balloons in clinical practice. Gastrointest Endosc Clin N Am 2017;27:245-256.

3. Yoo IK, Chun HJ, Jeen YT. Gastric perforation caused by an intragastric balloon: endoscopic findings. Clin Endosc 2017;50:602-604.

4. U.S. Food and Drug Administration. Liquid-filled intragastric balloon systems: letter to healthcare providers - potential risks [Internet]. Silver Spring (MD): U.S. Food and Drug Administration; c2017 [updated 2018 Jan 8; cited 2017 Oct 10]. Available from: https://www.fda.gov/Safety/ MedWatch/SafetyInformation/SafetyAlertsforHumanMedicalProducts/ ucm570916.htm.

5. Ponce J, Woodman G, Swain J, et al. The REDUCE pivotal trial: a prospective, randomized controlled pivotal trial of a dual intragastric balloon for the treatment of obesity. Surg Obes Relat Dis 2015;11:874-881.

6. Vyas D, Deshpande K, Pandya Y. Advances in endoscopic balloon therapy for weight loss and its limitations. World J Gastroenterol 2017;23:7813-7817.

7. ReShape Medical. ReShape integrated dual balloon system instructions for use [Internet]. San Clemente (CA): ReShape Medical; c2015 [updated 2015 Dec 15; cited 2017 Oct 10]. Available from: https://reshapeready. com/wp-content/uploads/2015/07/ReShape_Instructions_For_Use.pdf.

8. Al Shammari NM, Alshammari AS, Alkandari MA, Abdulsalam AJ. Migration of an intragastric balloon: a case report. Int J Surg Case Rep 2016;27:10-12. 